\title{
Characterization and Validation of a Novel Robotic System for Fluid-Mediated Programmable Stochastic Self-Assembly
}

\author{
Bahar Haghighat and Alcherio Martinoli
}

\begin{abstract}
Several self-assembly systems have been developed in recent years, where depending on the capabilities of the building blocks and the controlability of the environment, the assembly process is guided typically through either a fully centralized or a fully distributed control approach. In this work, we present a novel experimental system for studying the range of fully centralized to fully distributed control strategies. The system is built around the floating 3-cm-sized Lily robots, and comprises a water-filled tank with peripheral pumps, an overhead camera, an overhead projector, and a workstation capable of controlling the fluidic flow field, setting the ambient luminosity, communicating with the robots over radio, and visually tracking their trajectories. We carry out several experiments to characterize the system and validate its capabilities. First, a statistical analysis is conducted to show that the system is governed by reaction diffusion dynamics, and validate the applicability of the standard chemical kinetics modeling. Additionally, the natural tendency of the system for structure formation subject to different flow fields is investigated and corresponding implications on guiding the self-assembly process are discussed. Finally, two control approaches are studied: 1) a fully distributed control approach and 2) a distributed approach with additional central supervision exhibiting an improved performance. The formation time statistics are compared and a discussion on the generalization of the method is provided.
\end{abstract}

\section{INTRODUCTION}

Self-assembly (SA) plays a key role in many of the natural structuring phenomena at all scales. SA is defined as the reversible and spontaneous phenomenon of an ordered spatial structure emerging from the aggregate behavior of simpler preexisting building blocks through inherently local and random interactions in the system. The process of SA can be controlled through proper design of the building blocks, the environment, and the interplay of the involved forces. Several research efforts in the robotics community have been dedicated to development and study of experimental systems capable of realizing SA. The differences among these systems can be distinguished along three main axes: 1) the capabilities of the building blocks, 2) the controlability of the environment, and 3) the control approach, typically determined as a function of the former two.

While intelligent building blocks can actively take part in the SA process and thus allow for distributed control approaches [2], [3], [4], [5], the SA process of passive building blocks can only be controlled through a centralized approach [6], [7], [8]. Centralized control approaches become

Bahar Haghighat and Alcherio Martinoli are with the Distributed Intelligent Systems and Algorithms Laboratory, School of Architecture, Civil and Environmental Engineering, École Polytechnique Fédérale de Lausanne, Switzerland. firstname.lastnamedepfl.ch

This work has been sponsored by the Swiss National Science Foundation under the grant numbers 200021_137838/1 and 200020_157191/1.

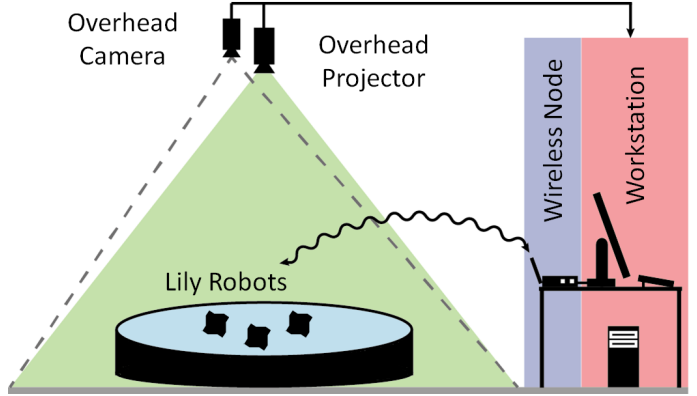

Fig. 1. An overview of the system: the experimental setup composed of a water-filled tank with peripheral pumps agitating the fluidic environment, overhead camera and projector, wireless node for establishing radio link between the workstation and the floating Lily robots [1].

quickly computationally intractable and unfeasible as the size of the swarm grows. Distributed control approaches on the other hand, offer the advantage of high scalability as they remain feasible for large swarms. An unexplored area of study is where a combination of distributed and centralized control approaches can be applied: the guided programmable SA of miniaturized robots, where the minimal design allows for realizing large swarms of intelligent modules at small scales while a controllable environment can additionally guide the SA process. This idea provides a promising means for programmable matter.

This work presents a novel experimental system for fluidmediated programmable stochastic SA of miniaturized robots (see Fig. 1). The system is an extension of our previous work in [6] which demonstrated fully centralized control of SA of passive modules, and is built around the intelligent Lily robots which have been specifically designed for operating in large swarms [1]. Lilies float on water and scavenge their motion from the ambient fluidic flow field. Their specific design allows for low-power latching and local communication through the same channel based on ElectroPermanent-Magnetic (EPM) technology, simplifying the robot internal design. They also communicate over radio with a base station. Being power-autonomous, Lilies can actively take part in a concurrent and parallel assembly process and report valuable detailed log information to the base station at all times. The robots can perceive the environment and obtain guidance from a central supervision either through specific radio commands or perceiving ambient luminosity. The setup built around the Lilies comprises a circular waterfilled tank with peripheral pumps, whereby the SA process is driven by the individually controllable flow of each pump. The dynamics of the system is imaged and tracked at a central 
station which also provides control of the pumps flow, wireless communication with the robots, and controlling the ambient luminosity. While the SA process towards a desired target structure can be mainly guided by the programmed behavior of the Lily robots in a fully distributed and thus scalable fashion, the environment can guide the process by adapting the flow field, changing the ambient luminosity pattern which can define regions or patches of different characteristics across the arena, and issue wireless inquiries or commands to the robots over radio. The versatile functionalities of our system allow for investigating a variety of control approaches. In this paper, we study programmable stochastic SA of 2D structures of floating Lily robots, leveraging our experimental setup. The aims of our study are 1) to characterize the system dynamics resulting from the interplay of the latching magnetic and the hydrodynamic forces acting on the robots, 2) to validate the capability of the system to accomplish SA tasks, and 3) to demonstrate different approaches for guiding the SA process.

\section{RELATED WORKS}

Several systems in the literature comprise intelligent and mobile building blocks. The cubic modules presented in [5] are capable of forming structures in $3 \mathrm{D}$, deploying magnetic latching and a lattice-based locomotion approach. These modules communicate over radio to a central supervisor which issues commands indicating the desired placement. SA of a swarm of fully autonomous and mobile floating robots in 2D has been studied in [9]. In addition to the floating robots, the system comprises a camera array for localizing the robots as well as a central computer which issues proper orders to the robots to assemble a specified structure.

Programmable SA employing a distributed control approach has been demonstrated in a large swarm of miniaturized mobile robots, the Kilobots [3]. Kilobots are specifically designed for experiments in large swarms. While the robots can be programmed wirelessly, they have no means to communicate back to the central computer. Despite the inherently noisy locomotion of the robots, a deterministic and quasi-serial structure formation scheme in 2D is obtained through a set of primitive collective behaviors followed by the robots. While this deterministic scheme allows for a reduced power consumption by the robots, since at each time only a few robots are moving, it slows down the convergence rate. In contrast, a stochastic scheme offers several benefits. Taking advantage of the stochastic ambient dynamics for module transportation allows for simplification of the internal design of the modules by reducing the on-board sensing, actuation, and power resources. The control approach (e.g., trajectory planning) and the necessary infra-structure (e.g., localization tools) are also simplified as a result.

Stochastic fluidic assembly of passive modules on an active substrate in 3D utilizing a central control approach is investigated in [7]. Floating Tribolons' stochastic assembly has been studied in [10], where the modules are only endowed with a single permanent magnet and actuated using a vibrating motor powered through a pantograph. The environment has no means to provide guidance to the process. As a result, the

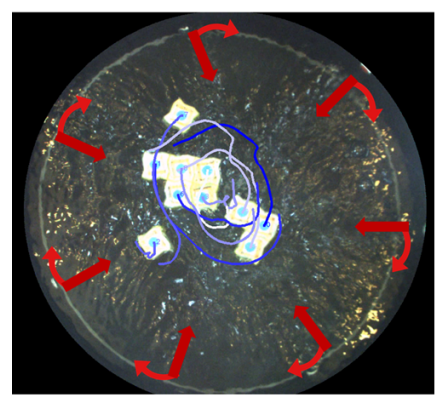

(a)

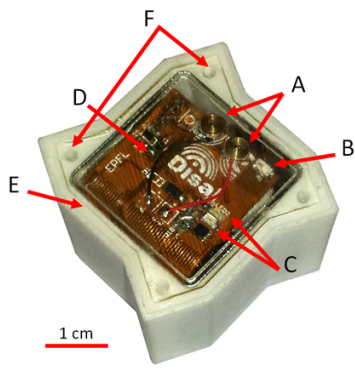

(b)
Fig. 2. (a) Visual tracking of 10 Lily robots during an experiment (the blue lines show a short trajectory history for each robot); (b) The Lily robot, key features visible in the picture are: charging contacts (A), chip antenna (B), two LEDs signaling board status (C), ambient light sensor (D), sealing gap filled with silicone paste (E), and two of the four trimming holes $(\mathrm{F})$.

obtained structures arise from the physics of the interactions between the building blocks and the forces acting in the system, and are thus fixed and not variable. The controlled SA of magnetically latching passive modules on water has been studied in a previous contribution of our laboratory [6]. The formation of target structures is modeled and controlled automatically and in real-time by a dedicated computational framework, which relies on a graphical description of the states and transitions in the system, resulting in scalability limitations for large swarms. Structure formation in the system of Pebble robots in [4] takes a different approach: the robots start in an ordered lattice, the stochastic forces in the environment are then relied upon to detach unwanted blocks. Pebbles are only powered when connected to the structure around a seed node connected to a power-supply. For sculpting the structure out of the initial lattice, proper messages issued from a central computer are propagated in the lattice. The intelligent modules in [2], which are capable of local communication through infra-red transceivers and controlling their permanent-magnet-based latches, stochastically selfassemble on an air table with controllable agitation modes, based on their internal behavior. While the environment can be controlled to allow for adapting the agitation modes of the air table, the modules have no means to perceive the environment or to communicate with a central supervisor during experiments.

\section{MATERIALS AND Methods}

In this section, we first explain our experimental system which consists of two main components: 1) the experimental setup built around the Lilies, and 2) the Lily robots which serve as the building blocks of the SA process. We then provide an overview of the SA scheme of the Lily robots, and detail the studied experimental scenarios.

\section{A. Experimental Setup}

The experimental setup consists of a circular water-filled tank equipped with peripheral pumps, an overhead camera, an overhead projector, a wireless node communicating with 
the robots, and a workstation. The Lily robots are not selflocomoted, they are instead stirred by the flow field produced by the pumps. The tank is approximately $1.2 \mathrm{~m}$ in diameter and $0.3 \mathrm{~m}$ in depth, and has seven inlets perpendicular to the wall which are endowed with a small insert piece to deviate the flow by about 15 degrees, creating a flow field with both radial and circular components. While the perpendicular flow components instigate irregular trajectories and induce collisions in the middle of the tank, they exhibit dead spots around the wall. The tangential components, however, generate a circular field, giving rise to regular closed trajectories which do not favor collisions but eliminate dead spots. To minimize any interference with the surface flow, the outlets are all placed at the bottom of the tank. Each pump's flow rate can be continuously controlled up to 9 $1 / \mathrm{min}$, allowing for a variety of flow fields and corresponding induced trajectories.

To monitor the evolution of the system, we use an overhead camera to track a passive marker located at the top of each robot using SwisTrack [11]. The positions of the markers are logged at a rate of approximately $30 \mathrm{~Hz}$. Complementary to the visual tracking data, is the data logged by the wireless node communicating with the robots over radio. These data contain the evolution of the robots' internal states. The wireless node is also used to program the robots and send commands or feedback to them during the experiment, enabling the robots to adapt their behavior according to the feedback which is based on a global image of the SA process rather than the robots' local perception.

The overhead projector allows for changing the ambient luminosity which is perceivable by the robots. Different ambient luminosity levels as well as patterns can be cast on the arena to define regions of different characteristics similar to [12]. As a result, while the information sent over radio can be used to adapt the behavior of uniquely identifiable robots, the lighting system can be used to adapt the behavior of the robots in specific regions of the arena.

\section{B. Lily Robots}

Lily robots, depicted in Fig. 2, are designed to function as the building blocks in our programmable stochastic SA system in large swarms [1]. Lilies are endowed with custom-designed EPM latches for latching and local communication. They can also communicate over a radio link to a base station to receive commands, new firmware, or to report specific information. Being powered by a small LiPo battery, Lilies can actively take part in the assembly process at all times.

Forming a target structure by a swarm of Lilies involves several aspects. Given a target structure, an appropriate behavioral ruleset is programmed on all robots through wireless bootloading. The robots EPM latches are by default enabled, resulting in a default latching upon meeting another robot. Once latched, the EPM-to-EPM inductive communication channel is physically established. The robots then exchange their internal states and look for an applicable rule in their ruleset. The ruleset contains interaction rules similar to chemical reactions, with the left-hand side representing the
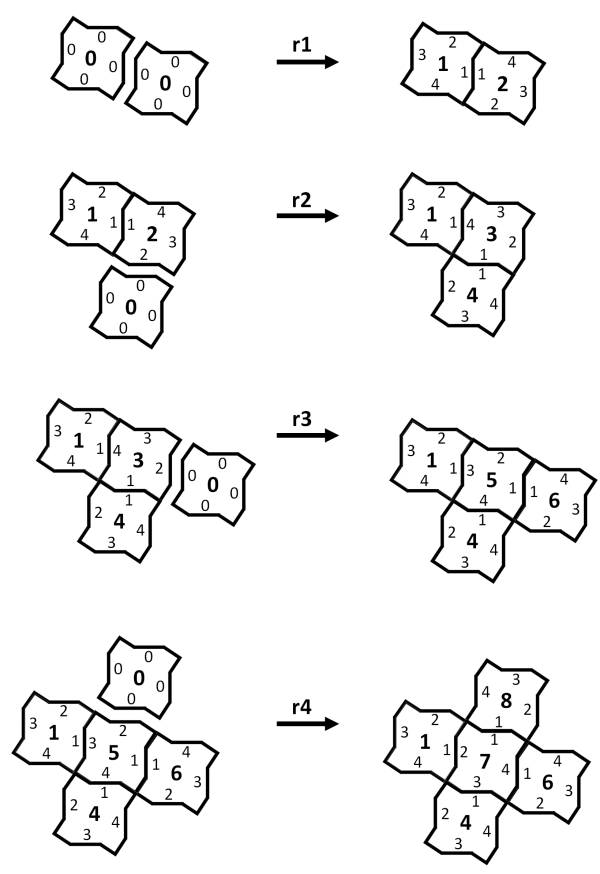

Fig. 3. Formation of a cross structure using $\phi_{\text {cross }}$ out of 5 Lily robots.

current self-state and the neighbor-state, and the right-hand side representing the corresponding updated states. If no applicable rule is found, the robots will unlatch by switching off their EPM latches; otherwise, they remain latched and update their internal states accordingly. Each Lily then updates the base station with its new internal state over the radio. This information can then be used to log the experiment and also as the ground truth for validating the models of the system. In addition to event-based reporting of their internal state, Lilies periodically communicate to the base station to check for pending commands such as a query about the battery voltage level or the internal state, a command for pausing the experiment, or a command for turning the robot off. This scheme allows the robots to spend most of their time in sleep mode or having the power-hungry radio transceiver off, thus resulting in an extended battery life. The commands from the base station can be as well used to modify the robots' behavioral ruleset on the fly.

\section{Self-assembly of Lily Robots}

We employ a dedicated software framework which allows for automatically synthesizing rulesets which are directly programmable on robotic modules [13]. The framework is based on an extended graph grammars formalism and generates rulesets based on a geometrical description of the desired target structure, a specified synthesis algorithm (for example the extended Singleton [13]), and the geometry of the robots. The internal state of a module is encoded using a pair $l=\left(l_{a}, l_{n}\right)$, denoted as an extended label. $l_{a}$ represents the control state of the robotic module and $l_{n}$ represents the index of the most recently engaged connector. We assume that the latching connectors are indexed following a counterclockwise $(\mathrm{CCW})$ rotation convention and that the robotic 
modules have a rotational symmetry. The synthesized rules describe interacting modules' states using a combination of a control state variable and a relative hop number. The idea is that the robotic modules can only take part in a reaction defined by a certain rule if they have the right control state and are participating in the reaction with the appropriate orientation. Once a latching connector is engaged, the robot communicates its internal state in the form of a relative extended label of $l=\left(l_{a}, l_{h}\right)$ with $l_{a}$ being the robot's control state and $l_{h}$ being a relative hop number which represents the relative orientation of the currently engaged connector with respect to its predecessor, assuming a CCW hop convention. For a module with an internal state of $\left(l_{a}, l_{n}\right)$ and $N$ connectors, $l_{h}=\left[\left(l_{n}-l_{c}\right) \bmod N\right]+1$, where $l_{n}$ and $l_{c}$ are the indexes of the most recently and the currently engaged connectors, respectively. For an isolated module the connectors are anonymous in terms of interaction possibilities, thus $l_{h}=0$. Assuming that Lilies are all initialized with a state of $(0,0)$, the ruleset below allows for formation of a cross shape as depicted in Fig. 3 The forward rules, $r_{1}$ to $r_{4}$, advance structure formation, while the backward rules, $\bar{r}_{1}$ to $\bar{r}_{4}$, allow for avoiding deadlocks [13].

$$
\phi_{\text {cross }}=\left\{\begin{array}{lllll}
(0,0) & (0,0) & \overrightarrow{r 1} & (1,1)-(2,1) \\
(0,0) & (2,2) & \overrightarrow{r 2} & (4,1)-(3,1) \\
(0,0) & (3,2) & \overrightarrow{r 3} & (6,1)-(5,1) \\
(0,0) & (5,2) & \overrightarrow{r 4} & (8,1)-(7,1) \\
(1,1)-(2,1) & \overrightarrow{\overline{r 1}} & (0,0) & (0,0) \\
(4,1)-(3,1) & \overrightarrow{\overline{r 2}} & (0,0) & (2,3) \\
(6,1)-(5,1) & \overrightarrow{\overline{r 3}} & (0,0) & (3,3) \\
(8,1)-(7,1) & \stackrel{\overline{r 4}}{\rightarrow} & (0,0) & (5,3)
\end{array}\right.
$$

\section{Experimental Scenarios}

In order to characterize the system and validate its functionalities, we conduct three main experiments. In experiment $\mathrm{A}$, the purpose is to assess the applicability of the standard chemical kinetics modeling to our system, and thus to show the relevance of our previous modeling efforts to the current experimental platform [6]. In experiment $\mathrm{B}$, we investigate the natural tendencies of the system to favor formation of certain structures depending on the latching forces and the flow field. In experiment $\mathrm{C}$, the formation of a cross shape structure is investigated. Two control approaches are used, a fully distributed control with a probabilistic ruleset where deadlock situations are avoided by setting proper non-zero backward rules probabilities, and a distributed control with a deterministic ruleset along with additional supervision through central control to avoid deadlocks.

\section{EXPERIMENTAL RESULTS}

Except for the last experiment using 5 robots, all the experiments were conducted using 10 Lily robots, and three main flow regimes were used. The flow regimes are referred to as high, medium, and low agitation modes hereafter, corresponding to $6.0 \mathrm{l} / \mathrm{min}, 4.4 \mathrm{l} / \mathrm{min}$, and $2.6 \mathrm{l} / \mathrm{min}$ flow rate for all pumps, respectively. To gather statistics, each experiment was repeated 10 times. The error envelopes denote one standard deviation interval around the mean value.

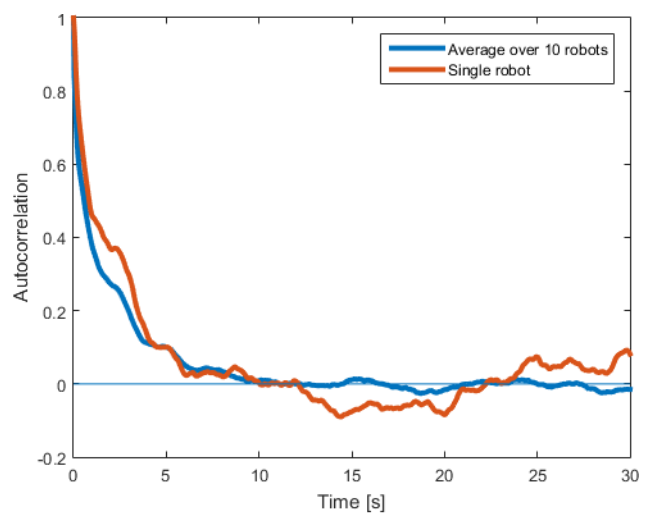

Fig. 4. Speed autocorrelation extracted from visual tracking of the robots.

\section{A. Macroscopic Kinetics}

Our previous work proposed a computational framework for automated modeling and control of SA [6]. The assumption of the framework was that the system was well-mixed and thus governed by reaction-diffusion dynamics, allowing us to apply canonical chemical kinetics models. Here we investigate the validity of such assumption for the current system.

1) Molecular Motion: For this experiment the robots were programmed with an empty ruleset, thus immediately detaching upon binding events. Diffusing particles exhibit the Brownian motion. A characteristic of such particles is that they quickly forget their speeds [2]. Fig. 4 shows the speed autocorrelation. The speed of each robot is essentially uncorrelated with its initial speed after approximately $5 \mathrm{~s}$. Additionally, we can quantify the mixing in the system. Diffusion provides for mixing in our system. For the system to be well-mixed it is required that the modules diffuse faster than they react. This can be quantitatively stated as $D / k>A$, where $D=0.0015 \mathrm{~m}^{2} / \mathrm{s}$ is the diffusion coefficient, $k=0.01$ $\mathrm{s}^{-1}$ is the typical reaction rate, and $A=0.09 \mathrm{~m}^{2}$ is the effective area in the system in presence of the high agitation mode. The diffusion coefficient for a module is estimated as the expected value of $r^{2}(t) / 4 t$, where $r(t)$ is the displacement of the module extracted from the visual tracking data.

\section{B. Natural Signature of the System}

While the SA process in our system is mainly guided by the programmed behavior of the Lily robots, the stochastic fluidic field is leveraged for bringing the robots into contact with one another. In our previous work with the passive Lily modules, we had noticed that each agitation mode in the system has its own signature distribution across the span of reachable assemblies. This was a result of the interplay of the magnetic latching force and the fluidic forces induced by the pumps' flow. The fact that the inter-module bonding was specifically designed to be reversible also allowed for a higher variability of observed assemblies for each agitation mode. In the current system with the Lily robots, the inter-robot bonds are designed to be more resilient, since the robots can always choose to turn their latches off if they wish to reject an interaction. In this experiment, we look at the growth rate of the size of the largest assembly in the system for the three 


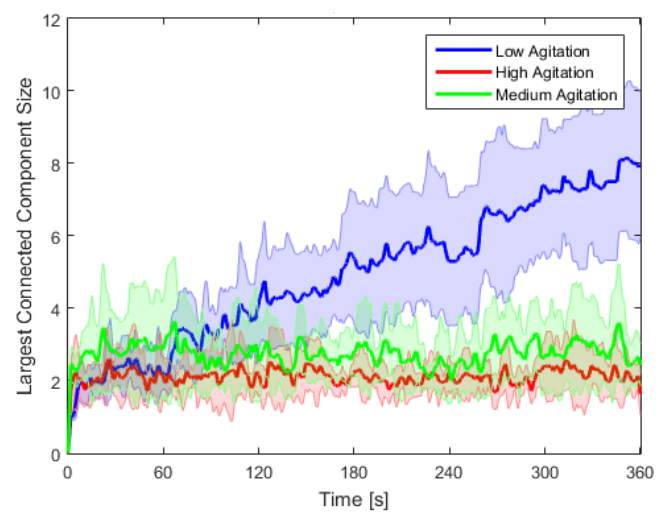

Fig. 5. Largest assembly size evolution, all robots have their EPMs off.

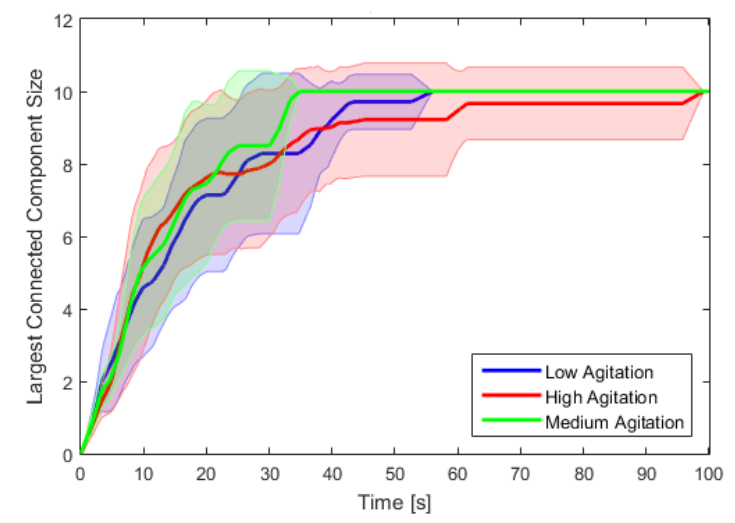

Fig. 6. Largest assembly size evolution, all robots have their EPMs on.

different aforementioned agitation modes, in two cases as depicted in Fig. 5 and Fig. 6 . While in the case where all of the robots have their EPMs on, higher energy agitations only slightly accelerate the growth of the assembly, when all EPMs are off, the final achievable size is strongly determined by the agitation mode. This shows the efficiency of the higher energy modes for taking the modules apart as a key factor in the unlatching mechanism. Furthermore, an interesting observation was made regarding the shape of the resulting structures when all robots have their EPM latches on: while for all studied agitation modes the system quickly ends up with one connected component, the resulting structures tend to have circular symmetry for the high agitation mode. On the other hand, chain-like structures are more common for the low agitation mode (see the supplementary video 1 ). This indicates that utilizing the higher agitation mode facilitates forming compact structures while the lower agitation mode allows the robots to form structures which span wider. This finding evidences the crucial role of the agitation modes in guiding the SA process. In other words, depending on whether the modules should be guided towards forming a compact structure or a widely spanning one, an appropriate agitation mode can be chosen similar to the approach employed in [6].

${ }^{1}$ The supplementary video is also available at:

\section{Cross-Shape Formation}

For these experiments, the robots were programmed with the $\phi_{\text {cross }}$ ruleset reported in Section III-C Two scenarios were studied. In the first scenario, the robots executed a deterministic ruleset. In order to avoid deadlock situations, the process was supervised by a human operator and proper orders were provided to the robots over radio. In particular, as soon as the system would end up in a state where two separate structures (i.e. the result of application of rules $\mathrm{r} 1$ and r2) were present and thus no further rule could be applied, a command over radio would be sent to order the two Lilies in the dipole structure to unlatch. In the second scenario, the robots executed a probabilistic ruleset and no central supervision or control was involved. For the probabilistic ruleset, all the forward rules probabilities were set to 1 , while for the backward rules probabilities of 0.05 for $\bar{r} 1,0.01$ for $\bar{r} 2$, and $\bar{r} 3$, and 0 for $\bar{r} 4$ were chosen. These values were empirically chosen based on the dynamics of the system, such that the average life time of the resulting structure from each forward rule (and destroyed by the corresponding backward rule) corresponds to the number of possibilities that the structure could further evolve towards the desired target of the cross shape. As a result, more complete structures would be more stable than the more primitive ones.

Fig. 7 shows the progress of the SA process for one run of the experiment with deterministic ruleset. The statistics of the total formation times are detailed in Table I. It can be seen that the average formation time of the deterministic ruleset coupled with the central supervision is slightly better than that of the probabilistic ruleset, with the standard deviation being significantly lower for the deterministic ruleset. These results support the idea that by involving a centralized supervision deadlocks can be avoided more efficiently, resulting in an overall improved formation time.

As briefly indicated earlier, using a fully centralized control approach can allow for exploiting the knowledge of the full state of the system in guiding the system towards the desired state. This can result in better performances compared to the case of fully distributed control where only local information is considered. However, this implies that the full weighted graph, representing the system states, as well as the transitions between them and their associated probabilities should be known. The knowledge about state transition probabilities can be obtained either through modeling or through direct observation and learning. The direct observation and learning as employed in [6], requires a long learning time which quickly grows with the number of states in the system. This is due to the fact that for the gathered statistics to be reliable, several observations should be obtained per transition. On the other hand, it is typically not easy to faithfully capture all the

TABLE I

\begin{tabular}{cccc}
\hline Ruleset & Control Approach & Mean & Std. \\
\hline Deterministic & Central supervision & $267.4 \mathrm{~s}$ & $103.8 \mathrm{~s}$ \\
Probabilistic & Fully distributed & $281.0 \mathrm{~s}$ & $150.7 \mathrm{~s}$ \\
\hline
\end{tabular}




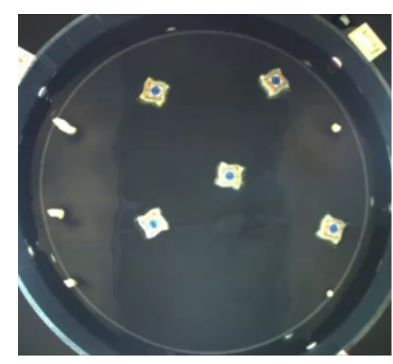

$0.0 \mathrm{~s}$

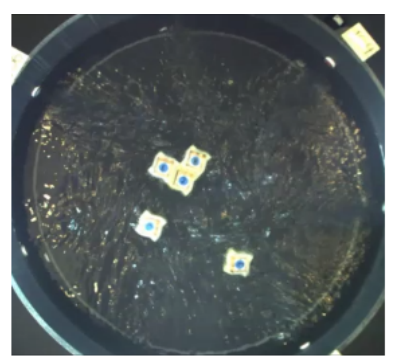

$29.0 \mathrm{~s}$

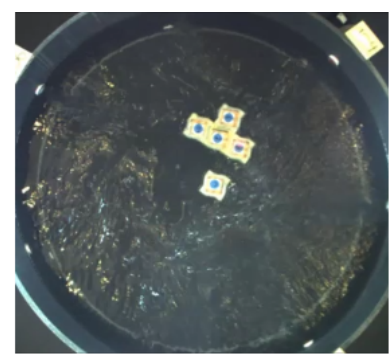

$101.0 \mathrm{~s}$

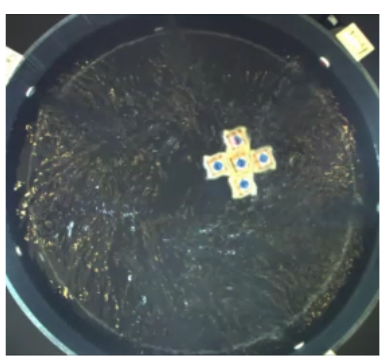

$120.1 \mathrm{~s}$

Fig. 7. A series of snapshots from the video data showing the formation of a cross shape structure by five Lily robots, executing a deterministic ruleset as explained in subsection IV-C (see also the video attachment).

physical phenomena in action in the system for the model to result in accurate transition probabilities estimations. As a result, a fully centralized control approach is typically restricted to systems of small swarm sizes or when the requirements on the formed structures are not very strict and thus the system graph can be reduced by lumping the states together (e.g., the shape of the structure can be ignored if only its cardinality is considered). However, another possibility is to let the assembly process to be guided in a distributed fashion by the programmed local behavior of the building blocks, while the central control is only employed to loosely guide and facilitate the convergence of the process. Since each ruleset behavior is equivalent to a certain path from the initial state towards the target state in the system graph, including supervision from a central supervisor which has the knowledge of the full state of the system can be used to adapt this path on the fly by issuing broadcast messages, or even by ordering specific robots to change their behavior, or by changing environmental characteristics (agitation and luminosity). Since the SA process is canalized through the behavior of the modules forming only specific structures, the restrictions on the central control of the environmental characteristics may be reduced significantly.

\section{CONClusion}

In this work, we have presented a novel experimental system for scalable programmable stochastic SA of floating miniaturized robots. The system is built around the Lily robots as the SA building blocks, and aims to provide a flexible platform for exploring the range of fully centralized to fully distributed control approaches for stochastic fluidic SA. Several experiments were carried out to characterize the dynamics of the system and validate its functionalities. Our findings evidenced that A) the system can be governed by reaction diffusion dynamics, B) different agitation modes in the fluidic arena exhibit essentially different characteristics which can be leveraged to shape structures, and that C) a centralized or supervised control can be leveraged to guide a distributedly controlled SA process in order to improve its efficiency. In the future, we plan to continue this work along several lines. In particular, we plan to leverage our experience with modeling distributed systems towards developing a unified scalable approach for modeling SA of modules endowed with a varying level of intelligence where a wide variety of control approaches can be applied. The system will be used to experimentally investigate multiple control algorithms for SA, in particular hybrid approaches, where a low-level distributed control is guided by a high-level central control, on a swarm of up to 100 Lily robots.

\section{REFERENCES}

[1] B. Haghighat, E. Droz, and A. Martinoli, "Lily: A miniature floating robotic platform for programmable stochastic self-assembly," in IEEE International Conference on Robotics and Automation, pp. 1941-1948, 2015.

[2] E. Klavins, "Programmable self-assembly," IEEE Control Systems, vol. 27, no. 4, pp. 43-56, 2007.

[3] M. Rubenstein, A. Cornejo, and R. Nagpal, "Programmable selfassembly in a thousand-robot swarm," Science, vol. 345, no. 6198, pp. 795-799, 2014.

[4] K. Gilpin, K. Koyanagi, and D. Rus, "Making self-disassembling objects with multiple components in the robot pebbles system," in IEEE International Conference on Robotics and Automation, pp. 36143621, 2011.

[5] J. W. Romanishin, K. Gilpin, S. Claici, and D. Rus, "3d m-blocks: Self-reconfiguring robots capable of locomotion via pivoting in three dimensions," in IEEE International Conference on Robotics and Automation, pp. 1925-1932, 2015.

[6] G. Mermoud, M. Mastrangeli, U. Upadhyay, and A. Martinoli, "Realtime automated modeling and control of self-assembling systems," in IEEE International Conference on Robotics and Automation, pp. 42664273, 2012.

[7] M. Tolley and H. Lipson, "Programmable 3d stochastic fluidic assembly of cm-scale modules," in IEEE/RSJ International Conference on Intelligent Robots and Systems, pp. 4366-4371, 2011.

[8] M. Mastrangeli, F. Schill, J. Goldowsky, H. Knapp, J. Brugger, and A. Martinoli, "Automated real-time control of fluidic self-assembly of microparticles," in IEEE International Conference on Robotics and Automation, pp. 5860-5865, 2014.

[9] I. O'Hara, J. Paulos, J. Davey, N. Eckenstein, N. Doshi, T. Tosun, J. Greco, J. Seo, M. Turpin, V. Kumar, et al., "Self-assembly of a swarm of autonomous boats into floating structures," in IEEE International Conference on Robotics and Automation, pp. 1234-1240, 2014.

[10] S. Miyashita, M. Kessler, and M. Lungarella, "How morphology affects self-assembly in a stochastic modular robot," in IEEE International Conference on Robotics and Automation, pp. 3533-3538, 2008.

[11] T. Lochmatter, P. Roduit, C. Cianci, N. Correll, J. Jacot, and A. Martinoli, "Swistrack-a flexible open source tracking software for multi-agent systems," in IEEE/RSJ International Conference on Intelligent Robots and Systems, pp. 4004-4010, 2008.

[12] N. Napp, S. Burden, and E. Klavins, "Setpoint regulation for stochastically interacting robots," Autonomous Robots, vol. 30, no. 1, pp. 57-71, 2011.

[13] B. Haghighat, B. Platerrier, L. Waegeli, and A. Martinoli, "Synthesizing rulesets for programmable robotic self-assembly: A case study using floating miniaturized robots," To appear in ANTS: International Conference on Swarm Intelligence, 2016. 